\title{
Papers
}

\section{Medical and psychosocial effects of early discharge after surgery for breast cancer: randomised trial}

\author{
Jorien Bonnema, Anneke M E A van Wersch, Albert N van Geel, Jean F A Pruyn, Paul I M Schmitz, \\ Marinus A Paul, Theo Wiggers
}

\begin{abstract}
Objective: To assess the medical and psychosocial effects of early hospital discharge after surgery for breast cancer on complication rate, patient satisfaction, and psychosocial outcomes.

Design: Randomised trial comparing discharge from hospital 4 days after surgery (with drain in situ) with discharge after drain removal (mean 9 days in hospital). Psychosocial measurements performed before surgery and 1 and 4 months after.

Setting: General hospital and cancer clinic in Rotterdam with a socioeconomically diverse population.
\end{abstract}

Subjects: 125 women with operable breast cancer.

Main outcome measures: Incidence of complications after surgery for breast cancer, patient satisfaction with treatment, and psychosocial effects of short stay or long stay in hospital.

Results: Patient satisfaction with the short stay in hospital was high; only 4\% (2/56 at 1 month after surgery and 2/52 at 4 months after surgery) of patients indicated that they would have preferred a longer stay. There were no significant differences in duration of drainage from the axilla between the short stay and long stay groups (median $8 v 9$ days respectively, $\mathrm{P}=0.45$ ) or the incidence of wound complications (10 patients $v 9$ patients). The median number of seroma aspirations per patient was higher for the long stay group ( $1 v 3.5, \mathrm{P}=0.04)$. Leakage along the drain occurred more frequently in short stay patients (21 $v 10$ patients, $\mathrm{P}=0.04$ ). The two groups did not differ in scores for psychosocial problems (uncertainty, anxiety, loneliness, disturbed sleep, loss of control, threat to self esteem), physical or psychological complaints, or in the coping strategies used. Before surgery, short stay patients scored higher on scales of depression $(\mathrm{P}=0.03)$ and after surgery they were more likely to discuss their disease with their families (at 1 month $\mathrm{P}=0.004$, at 4 months $\mathrm{P}=0.04$ ).

Conclusions: Early discharge from hospital after surgery for breast cancer is safe and is well received by patients. Early discharge seems to enhance the opportunity for social support within the family.

\section{Introduction}

The length of time patients spend in hospital after surgical procedures has been decreasing. ${ }^{12}$ Patients having surgery for breast cancer are considered especially suitable for shorter stays in hospital because recovery after surgery is usually rapid. These patients usually remain in hospital for 9 to 12 days, until the serous fluid produced by the axilla is minimal and the closed suction drain is removed. ${ }^{3}$ Shorter hospital stays are possible if patients are discharged with their drains in situ ${ }^{4}$ or if drains are removed early. ${ }^{5}$ Several studies have claimed that these procedures are safe. ${ }^{4-8}$ However, these studies have been retrospective, ${ }^{6}$ have given little information about the selection of controls, ${ }^{45}$ or have used self selected patients. ${ }^{8}$ These factors make the results difficult to interpret.

Patient satisfaction with early discharge is reported to be high. ${ }^{4-9}$ Recovery in the patient's own environment may result in better psychosocial adjustment as a result of enhanced patient comfort, control, independence, and better interaction with family members. ${ }^{10}$ In the only study of the psychological effects of early discharge, no adverse effects were found, but patients in this study decided for themselves that they would leave hospital early. ${ }^{8}$

We conducted a randomised trial to compare short and long postoperative stays in hospital after surgery for breast cancer to determine the effect of early discharge on complication rate, patient satisfaction, and psychosocial outcome. We hypothesised that there would be no differences between the two interventions.

\section{Subjects and methods}

\section{Patients}

Patients were eligible for inclusion in the study if they had stage I or II breast cancer, had been referred to the Daniel den Hoed Cancer Center and Zuider hospital, and had been selected for treatment by either modified radical mastectomy or lumpectomy with axillary dissection. Patients were excluded if they had received preoperative radiotherapy or chemotherapy, were at high risk of complications (category III or higher of the American Society of Anesthesiologists classification), or were mentally incompetent; patients who had difficulties with the Dutch language or an inappropriate home situation were also excluded.

\author{
Department of \\ Surgical Oncology, \\ University Hospital \\ Rotterdam/Daniel \\ den Hoed Cancer \\ Center, Zuider \\ Hospital \\ Rotterdam, PO Box \\ $5201,3008 \mathrm{AE}$ \\ Rotterdam, \\ Netherlands \\ Jorien Bonnema, \\ surgeon \\ Albert N van Geel, \\ surgeon \\ Paul I M Schmitz \\ statistician \\ Marinus A Paul, \\ surgeon \\ Theo Wiggers, \\ surgeon \\ Institute for Health \\ and Environmental \\ Issues, PO Box 71, \\ $4797 \mathrm{ZH}$ \\ Willemstad, \\ Netherlands \\ Anneke M E A van \\ Wersch, \\ social psychologist \\ Jean F A Pruyn, \\ social psychologist \\ Correspondence to: \\ Mrs Bonnema \\ wiggers@chih.azr.nl
}

BMJ 1998;316:1267-71

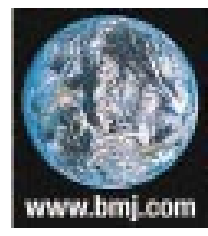

Further data are available on the internet 
Between October 1993 and April 1995, 139 out of $173(80 \%)$ women with operable breast cancer were enrolled in the study: 69 were assigned to short stay treatment and 70 to long stay treatment. Women randomised to short stay treatment were discharged on the morning of the fourth day after surgery with the axillary drain in situ. Women randomised to long stay treatment were discharged after their drain had been removed.

Of the 34 women who were not entered into the study, 22 declined to participate, 10 had an unsatisfactory home situation, and two were not asked to participate. Fourteen more women were excluded after randomisation: two long stay patients received preoperative chemotherapy, one long stay patient was treated in another hospital, one short stay patient had no malignancy, and 10 patients withdrew from the study. Reasons given for short stay patients withdrawing from the study were: questionnaires too difficult (2), refusing home care (2), dissatisfaction with randomisation outcome (1), and unknown reason (1). Reasons for long stay patients withdrawing from the study were: dissatisfaction with randomisation outcome (1), unwillingness to fill out forms (2), and unknown reason (1). Thus, the final group consisted of 125 patients: 62 short stay and 63 long stay.

\section{Randomisation and study design}

Approval from the ethics committees of both hospitals was obtained before the start of the study. Written informed consent was obtained from all patients.

A randomisation list was prepared by the statistician (PIMS) using a program for the generation of random numbers and assignment into two groups with a prespecified size of blocks. The size of the blocks (8 patients) was not known by the investigators, and no stratification was applied. The randomisation list was accessible only to the data managers of the central trial office at the Daniel den Hoed Cancer Center. The patient was informed of her diagnosis, treatment plan, and the design of the study by her surgeon. The patient's home situation was subsequently assessed by a breast cancer nurse. Surgeons telephoned the trial office to discover each eligible patient's randomisation before admission.

An early discharge protocol was developed to guarantee continuity of care. It included structured patient education provided by the breast cancer nurse and also available in written form, referral to a community health nurse, provision of an emergency telephone number, the scheduling of follow up visits, and an information letter being sent to the general practitioner. The development and implementation of this protocol have been described. ${ }^{11}$ For women assigned to short stay treatment, drain removal was performed at home or in the outpatient clinic. For both groups drains were removed when the production of serous fluid was less than $30 \mathrm{ml}$ per day or after 14 days. Nursing care of the wound and drain, and the provision of arm exercises, protheses, and psychosocial guidance were standardised for both groups.

Patients were followed up for 4 months. At admission, patients were given a daily diary, to be used for one month, and a weekly diary, to be used for the following 3 months. The length of stay in hospital was recorded in the diaries. Clinical study end points were recorded in the diaries and patients' files by the doctors and nurses.

Three questionnaires were used to assess psychosocial variables and record demographic characteristics. The first was distributed at admission and completed the same day; the second questionnaire was distributed 1 month after surgery, and the third 3 months later, during outpatient visits.

\section{Study end points}

\section{Complications}

Complications recorded included infection, necrosis, haematoma, and dehiscence. Wound infection was defined according to the standards of the Centers for Disease Control and Prevention. ${ }^{12}$ Necrosis was defined as any visible necrosis along the edge of the wound. Blood that had collected under the skin, and that was removed by puncture or opening of the wound, was considered to be a haematoma. Drain complications were also recorded. After the drain was removed, fluid collection in the axilla that was clinically apparent was defined as seroma and removed by percutaneous aspiration.

\section{Patient satisfaction}

Patient satisfaction with the length of stay was assessed with questions about preferences for a shorter or longer stay. Patients were also asked if they would recommend short stay treatment to other patients. Satisfaction with the care provided by the community health nurse was also assessed.

\section{Psychosocial variables}

The psychosocial functioning of patients was evaluated using validated scales based on a theoretical model of coping with cancer developed by van den Borne and Pruyn. ${ }^{13}$ Some specific items concerning breast cancer were added. Scale structures were made by factor analyses and were similar to those found in previous research. ${ }^{14}$ The reliability indices of the scales, assessed for each of the three questionnaires, were evaluated using Cronbach's $\alpha .^{15}$ Scores varied between 0.62 and 0.95 with most $>0.70$. Three out of 57 scores were excluded from analysis because the reliability of the scale was too low $(\alpha<0.60)$.

The following variables were measured: uncertainty, ${ }^{14}{ }^{16-18}$ state and trait anxiety, ${ }^{19}$ object anxiety, ${ }^{14} 16-18$ loneliness, ${ }^{14}{ }^{16-18}$ depression, ${ }^{14}{ }^{16-18}$ sleep disturbances, ${ }^{14}{ }^{18}$ feelings of loss of control, ${ }^{14} 1618$ self esteem,,${ }^{14}{ }^{18}$ and the cancer locus of control. ${ }^{20}$ Locus of control refers to whether patients attribute the cause of their cancer to personal or situational factors. The Rotterdam symptom checklist was used to assess physical and psychosocial complaints. ${ }^{21}$

Coping strategies were assessed with scales constructed previously. ${ }^{14}$ Communication about the disease in the home was evaluated with a scale that assesses the openness of discussion within the family, with the patient's partner, and with the patient's children. ${ }^{17}$

\section{Statistical considerations}

A primary objective in this trial was to calculate a degree of patient satisfaction in the short stay group that would be about equal to the satisfaction found in 
long stay patients. We hypothesised that at 1 month after surgery, $5 \%$ of long stay patients at most would have preferred a longer stay in hospital. We also supposed that if the percentage of patients satisfied with their stay in hospital was equal the upper $95 \%$ confidence limit for the difference in satisfaction should not exceed $10 \%$ with a probability of $80 \%$ $\left(\alpha=5 \%\right.$ one tailed, $\left.\beta=20 \%{ }^{22}\right)$. For these specifications $2 \times 57=114$ patients were necessary. To allow for withdrawals we decided to randomly allocate interventions to $140-150$ patients.

For the 125 patients who were studied the power for comparing several outcomes can be calculated (all comparisons with $\alpha=0.05$ ). The statistical power was $99 \%$ (SD $400 \mathrm{ml}$ within groups) for detecting a difference of $300 \mathrm{ml}$ in total volume of axillary drainage between the groups. A difference between groups in the duration of axillary drainage of 1.5 days was detectable with a power of $80 \%$ (SD 3 days within groups). The sample size was inadequate to detect small but clinically significant wound complications ( $5 \%$, power about $50 \%$ ).

\section{Data analysis}

Psychosocial variables were analysed with the SPSS package. All other analyses were performed using STATA release 5.0 (StatCorp, College Station, TX). The $\chi^{2}$ test was used to compare data between categories without correction for continuity. Fisher's test of exact probability was applied in $2 \times 2$ tables with small expected numbers. Student's $t$ test was used to analyse continuous variables in the psychosocial part of the study. The Mann-Whitney U test was used to compare data on drainage between the two groups. Significance was defined as $\mathrm{P}<0.05$.

\section{Results}

The two groups were comparable in tumour stage, type of treatment, age, marital status, family income, and educational level (data available on the internet at www.bmj.com). Women in the short stay group were in hospital a median of 4 days (mean 4.1 including day of discharge, range 3-5); women in the long stay group had a median length of stay of 9 days (mean 9.0 including day of discharge, range 4-14).

\section{Complications}

There were no significant differences between short stay and long stay patients in drainage volume or duration of drainage, but the mean number of aspirations required per patient was higher in the long stay group $(\mathrm{P}=0.04) \quad$ (table 1). Clinically significant wound infection occurred in eight patients in the short stay group and in seven patients in the long stay group; all were treated with antibiotics. One short stay and two long stay patients also required abscess drainage. Two short stay patients were readmitted for removal of a persistent haematoma. Leakage of drainage fluid alongside the drain occurred more often in the short stay group (in $21 v 10$ patients, $\mathrm{P}=0.04$ ). One short stay patient died of unsuspected distant metastases during the study.

\section{Patient satisfaction}

Table 2 shows patients' satisfaction with their length of stay. Most of the women in the short stay group
Table 1 Complications among patients after surgery for breast cancer according to length of stay in hospital

Short stay $(n=61)^{\star} \quad$ Long stay $(n=59) \dagger \quad P$ value

\begin{tabular}{|c|c|c|c|}
\hline & 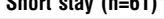 & Leng ong & Forio \\
\hline \multicolumn{4}{|l|}{ Drainage } \\
\hline \multicolumn{4}{|l|}{ Median (range) total volume $(\mathrm{ml})$ : } \\
\hline From axillary drain & $515(400-3000)$ & $685(30-2130)$ & 0.19 \\
\hline From drain in breast wound & $175(5-885)$ & $80(10-1070)$ & 0.51 \\
\hline \multicolumn{4}{|l|}{ Duration (days): } \\
\hline From axillary drain & $8(1-15)$ & $9(2-14)$ & 0.45 \\
\hline From drain in breast wound & $3(1-12)$ & $2(1-9)$ & 0.27 \\
\hline \multicolumn{4}{|l|}{ Aspiration } \\
\hline No (\%) of patients who had aspiration & $10(16)$ & $8(14)$ & 0.80 \\
\hline Median No (range) aspirations per patient & $1(1-3)$ & $3.5(1-7)$ & 0.04 \\
\hline Median (range) total volume aspirated (ml) & $105(5-650)$ & $400(150-880)$ & 0.01 \\
\hline \multicolumn{4}{|l|}{ Wound complications } \\
\hline \multicolumn{4}{|l|}{ No (\%) of patients with: } \\
\hline Haematoma & $2(3)$ & $1(2)$ & 1.00 \\
\hline Necrosis & 0 & $1(2)$ & 0.49 \\
\hline Infection & $8(13)$ & $7(12)$ & 1.00 \\
\hline Dehiscence & $1(2)$ & $1(2)$ & 1.00 \\
\hline Any type of wound complication & $10(16)$ & $9(15)$ & 1.00 \\
\hline \multicolumn{4}{|l|}{ Drainage complications } \\
\hline \multicolumn{4}{|l|}{ No (\%) of patients with: } \\
\hline Obstruction & $20(33)$ & $15(25)$ & 0.42 \\
\hline Loss of vacuum & $24(39)$ & $16(27)$ & 0.18 \\
\hline Leakage & $21(34)$ & $10(17)$ & 0.04 \\
\hline Loss of drain & $5(8)$ & $2(3)$ & 0.44 \\
\hline Any type of drain complication & $38(62)$ & $27(46)$ & 0.10 \\
\hline
\end{tabular}

*Discharged 4 days after surgery.

$\dagger$ Discharged after drain removal (median 9 days after surgery).

Table 2 Patient satisfaction with short stay or long stay in hospital after surgery for breast cancer. Values are numbers (percentages) of patients

\begin{tabular}{|c|c|c|c|c|}
\hline & $\begin{array}{l}\text { Short stay } \\
(n=62)^{\star}\end{array}$ & $\begin{array}{c}\text { Long stay } \\
(n=63) \dagger\end{array}$ & $\begin{array}{l}\text { Mean difference } \\
(\%)(95 \% \mathrm{CI})\end{array}$ & $P$ value \\
\hline \multicolumn{5}{|c|}{ Patient would have preferred longer hospital stay: } \\
\hline 1 month after surgery & $2 / 56(4)$ & $7 / 52(14)$ & $-10(-20$ to 0.6$)$ & 0.08 \\
\hline 4 months after surgery & $2 / 52(4)$ & $4 / 44(9)$ & $-5(-15$ to 5$)$ & 0.41 \\
\hline \multicolumn{5}{|c|}{ Patient would have preferred shorter hospital stay: } \\
\hline 1 month after surgery & $8 / 55(15)$ & $16 / 53(30)$ & $-16(-31$ to -0.2$)$ & 0.05 \\
\hline 4 months after surgery & $7 / 51(14)$ & $15 / 46(33)$ & $-19(-35$ to -2$)$ & 0.03 \\
\hline \multicolumn{5}{|c|}{ Patient would recommend short stay to other patients: } \\
\hline 1 month after surgery & $51 / 55(93)$ & $17 / 46(37)$ & 67 (40 to 71$)$ & $<0.001$ \\
\hline 4 months after surgery & $50 / 52(96)$ & $19 / 45(42)$ & 54 (39 to 69$)$ & $<0.001$ \\
\hline
\end{tabular}

*Discharged 4 days after surgery.

†Discharged after drain removal (median 9 days after surgery).

indicated that they would recommend early discharge to other patients, as did $37 \%$ of the long stay patients at 1 month and $42 \%$ of long stay patients at 4 months, despite the fact that they had no experience of early discharge (table 2).

Evaluation of the nursing care provided at home showed that 42 out of $45(93 \%)$ short stay patients were satisfied that they had received enough attention and that 30 out of $42(71 \%)$ felt as secure at home as in hospital.

\section{Psychosocial variables}

There was no difference between the two groups in scores on scales measuring uncertainty, anxiety, loneliness, disturbed sleep, loss of control, or threats to self esteem. Before surgery short stay patients scored higher than long stay patients on scales measuring depression (score $10.3 v 8.9, \mathrm{P}=0.03$; minimum score 6 , maximum score 24). ${ }^{14}{ }^{18}$ This difference disappeared after surgery. There were no differences in physical or 
psychological complaints, as measured by the Rotterdam symptom checklist, or in coping strategies used.

A shorter stay in hospital seemed to influence the extent to which the disease could be discussed within the patient's family. Before surgery there were no differences between the two groups, but at 1 and 4 months after surgery short stay patients were more likely to discuss their disease with their family (score 1 month after surgery $23.2 \vee 21.5, \mathrm{P}=0.004$; score 4 months after surgery $23.5 \approx 21.9, \mathrm{P}=0.04$; minimum score 7 , maximum score 28$){ }^{17}$

\section{Discussion}

This paper presents the results of a randomised trial evaluating the medical and psychosocial effects of short and long hospital stays after surgery for breast cancer. Comparison between the two groups found no significant differences in wound complications, duration of drainage, patient satisfaction, or psychosocial outcomes. In fact there seemed to be an increase in social support within the family among patients in the short stay group.

The high scores for treatment satisfaction among the short stay patients are in accordance with the results of other studies. ${ }^{4-9}$ Short stay patients were highly satisfied with their community based nursing care. Support from a specialist nurse considerably reduces psychological morbidity. ${ }^{23}$ In the home, community nurses take on the role of breast cancer nurses. We considered it important to continue this care after a short stay in hospital.

There were no adverse effects of a shorter stay in hospital on the rate of complications or the incidence of seroma formation. However, the number of patients in this study was too small to detect a difference of $5 \%$ in rates of wound complication; a sample size of more than 800 patients would have been necessary to do this. This is not feasible in this type of research. We decided to discharge patients with drains in situ and to remove drains when production of serous fluid was minimal. This practice leads to a low incidence of seroma aspiration ${ }^{24}{ }^{25}$ and fewer outpatient visits. The alternatives are to remove the drain after a fixed number of days regardless of fluid production ${ }^{526} 27$ or not to place a drain in the axilla. ${ }^{27}{ }^{28}$ Seromas have been reported in as few as $10 \%$ of patients after early drain removal, ${ }^{5}$ but others have reported seromas in as many as $40 \%{ }^{3}$ and $73 \%{ }^{27}$ of patients, though these did not affect the risk of infection. The length of time the drain was in situ was equal for both groups and is consistent with previous findings from our own clinic. ${ }^{29}$

Before surgery the patients randomly allocated to a short hospital stay scored higher on scales measuring depression than did those randomly allocated to a long stay. The uncertainty about the experimental treatment after surgery may have contributed to these feelings. A shorter stay in hospital seems to make it easier for a patient to discuss the disease with her family; however, the data should be interpreted carefully as this was the only significant difference in psychosocial variables found between the two groups after surgery. The positive effects of social support in psychosocial adjustment for patients with breast cancer have been discussed. ${ }^{30} 31$ The ability to express emotions within the family is associated with less mood disturbance. ${ }^{32}$ In our study
- Early discharge from hospital after breast cancer surgery does not lead to an increase in the incidence of wound infection or seroma formation

- A short stay in hospital, with support from community nurses on the patient's return home, is acceptable to patients

- Psychosocial rehabilitation is not influenced by early discharge

- Recovery in the family environment may facilitate discussion of the illness

- Patients recovering from surgery for breast cancer need not spend more than three days in hospital provided that they are in good physical condition and there is adequate nursing support available in the community

there was no decrease in mood disturbance in the short stay group; our follow up was 4 months, but the positive effects may have become evident later.

In the United States patients having surgery for breast cancer often stay in hospital only one or two days $^{4}{ }^{10}$ or are treated as outpatients. ${ }^{6}$ These changes were initially financially motivated but have gradually become accepted by surgeons. ${ }^{10}$ In most European hospitals, however, these types of early discharge policies are not the normal practice. Our randomised study has proved that shortening the length of time a patient spends in hospital after surgery for breast cancer has no adverse effects. It would be interesting to evaluate the American practice in a European setting, paying special attention to the psychosocial effects of this policy, especially since no data have been available on these aspects until now.

We thank all participants who enrolled in the trial and those who contributed and are not mentioned here. We thank $\mathrm{P}$ Stringer and A M M Eggermont for reading the manuscript.

Contributors: JB contributed to the design of the protocol, performed the literature search, participated in the execution of the study, collected and analysed the data, wrote the paper, and is guarantor for the study. AMEAvW coordinated the study in both hospitals, discussed core ideas, studied the literature, participated in data collection and analysis, and contributed to writing the paper. JFAP initiated and coordinated the formulation of the study hypothesis, designed the protocol, contributed to the interpretation of findings, and edited the paper. PIMS contributed to the design of the protocol, coordinated randomisation procedures, and performed the statistical analysis of the data. MAP contributed to data collection and editing the paper. TW had the original idea for the study, initiated the research, participated in the execution of the study, and edited the paper.

Funding: Ministry of Welfare, Health, and Sports, the Netherlands.

Conflict of interest: None.

1 McAleese P, Odling-Smee W. The effect of complications on length of stay. Ann Surg 1994;220:740-4.

2 Maddern GJ. The changing pattern of surgery. BrJ Surg 1996;83:145-6.

3 Inwang R, Hamed H, Chaudary MA, Fentiman IS. A controlled trial of short-term versus standard axillary drainage after axillary clearance and Short-term versus standard axillary drainage after axillary clearance and
iridium implant treatment of early breast cancer. Ann R Coll Surg Engl 1991;73:326-8.

4 Clark JA, Kent RB. One-day hospitalization following modified radical mastectomy. Am Surg 1992;58:239-42.

5 Yii M, Murphy C, Orr N. Early removal of drains and discharge of breast cancer surgery patients: a controlled prospective clinical trial. Ann R Coll Surg Engl 1995;77:377-9. 
6 Goodman AA, Mendez AL. Definitive surgery for breast cance performed on an outpatient basis. Arch Surg 1993;128:1149-52.

7 Holcombe C, West N, Mansel RE, Horgan K. The satisfaction and savings of early discharge with drain in situ following axillary lymphadenectomy in the treatment of breast cancer. Eur J Surg Oncol 1995;21:604-6.

8 Boman L, Björvell H, Cedermark B, Theve NO, Wilking N. Effects of early discharge from hospital after surgery for primary breast cancer. Eur J Surg 1993;159:67-73.

9 Pedersen SH, Douville LM, Eberlein TJ. Accelerated surgical stay programs. A mechanism to reduce health costs. Ann Surg 1994;219:37481.

10 Kambouris A. Physical, psychological, and economic advantages of accelerated discharge after surgical treatment for breast cancer. Am Surg 1996;62:123-7.

11 Van Wersch AMEA, Bonnema J, van Geel AN, Prinsen B, Pruyn JFA, Wiggers T. Continuity of information for breast cancer patients: the development, use and evaluation of a multidisciplinary care protocol. Patient Education and Counseling 1997;30:175-86.

12 Garner JS, Jarvis WR, Emori TG, Horan TC, Hughes JM. CDC definitions for nosocomial infections, 1988. Am J Inf Control 1988;16:128-40.

13 Pruyn JFA. Coping with stress in cancer patients. Patient Education and Counseling 1983;5:57-62.

14 Van den Borne HW, Pruyn JFA. Lotgenotencontact bij kankerpatiënten. [Contacts between fellow cancer patients.] Maastricht: van Gorcum, 1985

15 Cronbach LJ. Essentials of psychological testing. New York: Harper and Row, 1990.

16 De Boer MF, Pruyn JFA, van den Borne HW, Knegt PP, Ryckman RM, Verwoerd CDA. Rehabilitation outcomes of long-term survivors treated for head and neck cancer. Head Neck 1995;17:503-15.

17 Mesters I, van den Borne HW, McCormick L, Pruyn JFA, de Boer MF, Imbos I. Openness to discuss cancer in the nuclear family scale: development and validation. Psychosom Med 1997;59:269-79.

18 Van den Borne HW, Pruyn JFA, van den Heuvel WJA. Effects of contacts between cancer patients on their psychosocial problems. Patient Education and Counseling 1987;9:33-51.

19 Spielberger CD, Gorsuch RL, Lushene RE. STAI manual for the state-trait anxiety inventory. Palo Alto: Consulting Psychologists Press, 1970
20 Watson M, Greer S, Pruyn JFA, van den Borne HW. Locus of control and adjustment to cancer. Psychol Rep 1990;66:39-48.

21 De Haes JCJM, van Knippenberg FCE, Neijt JP. Measuring psychological and physical distress in cancer patients: structure and application of the Rotterdam Symptom Checklist. Br J Cancer 1990;62:1034-8.

22 Makuch R, Simon R. Sample size requirements for evaluating conservative therapy. Cancer Treat Rep 1978;62:1037-40.

23 McArdle JMC, George WD, McArdle CS, Smith DC, Moodie AR, Hughson AVM, et al. Psychological support for patients undergoing breast cancer surgery: a randomised study. BMJ 1996;312:813-6.

24 Tadych K, Donegan WL. Postmastectomy seromas and wound drainage. Surg Gynecol Obstet 1987;165:483-7.

25 Vinton AL, Traverso LW, Jolly PC. Wound complications after modified radical mastectomy compared with tylectomy with axillary lymph node dissection. Am J Surg 1991;161:584-8.

26 Coveney EC, O'Dwyer PJ, Geraghty JG, O'Higgins NJ. Effect of closing dead space on seroma formation after mastectomy. A prospective randomized clinical trial. Eur J Surg Oncol 1993;19:143-6.

27 Somers RG, Jablon LK, Kaplan MJ, Sandler GL, Rosenblatt NK. The use of closed suction drainage after lumpectomy and axillary node dissection for breast cancer. Ann Surg 1992;215:146-9.

28 Jeffrey SS, Goodson WH, Ikeda DM, Birdwell RL, Bogetz MS. Axillary lymphadenectomy for breast cancer without axillary drainage. Arch Surg 1995;130:909-13.

29 Bonnema J, van Geel AN, Ligtenstein DA, Schmitz PIM, Wiggers T. A prospective randomized trial of high versus low vacuum drainage after axillary dissection for breast cancer. Am J Surg 1997;173:76-9.

30 Nelles WB, McCaffrey RJ, Blanchard CG, Ruckdeschel JC. Social support and breast cancer: a review.J Psychosoc Oncol 1991;9:21-35.

31 Bloom JR. Social support, accommodation to stress and adjustment to breast cancer. Soc Sci Med 1982;16:1329-38.

32 Spiegel D, Bloom JR, Gottheil E. Family environment as a predictor of adjustment to metastatic breast carcinoma. J Psychosoc Oncol 1983;1: 33-44.

(Accepted 22 October 1997)

\title{
Resolution of peanut allergy: case-control study
}

\author{
Jonathan O’B Hourihane, Stephen A Roberts, John O Warner
}

\begin{abstract}
Objectives: To determine whether there are any differences between children who remain mildly or moderately allergic to peanut and children with similar histories but a negative reaction on challenge with peanut.
\end{abstract}

Design: Case-controls matched for age and sex. Setting: Children's day wards in two teaching hospitals.

Intervention: Open food challenge with peanut. Subjects: 15 children with resolved peanut allergy (resolvers) and 15 with persistent allergy (persisters). Main outcome measure: Reaction on challenge with peanut, serum total and peanut specific IgE concentrations.

Results: The groups had a similar median age at first reaction to peanut (11 months, range 5-38) and similar symptoms. Allergy to other foods was less common in resolvers $(2 / 15)$ than persisters $(9 / 15)$ $(\mathrm{P}=0.02)$. On skin prick testing with peanut all 13 resolvers tested but only $3 / 14$ persisters had a weal of $<6 \mathrm{~mm}(\mathrm{P}<0.0001)$. Total and peanut specific $\operatorname{IgE}$ concentrations did not differ much between the groups.

Conclusion: Appropriately trained clinicians must be prepared to challenge preschool children with peanut as some will be tolerant despite a history of reactions to peanut and a positive skin prick test with peanut. Preschool children whose apparent peanut allergy is refuted by food challenge show allergy to other foods less often than those in whom peanut allergy persists. The size of weal on skin prick testing to peanut predicts reactivity but not severity on peanut challenge.

\section{Introduction}

The diagnosis of peanut allergy has important consequences for patients and their families. They are told that allergic reactions occur after frequent exposure, that reactions are often severe, and that the allergy persists indefinitely.

The dietary habits of the British population have changed, with vegetarianism becoming more popular and the use of peanut butter apparently increasing as a snack food for children. These changes may be linked to a recently observed decrease in the age of onset of peanut allergy. ${ }^{3}$

In longitudinal studies allergies to cows' milk and egg usually resolve early in life; $85 \%$ of children with cows' milk allergy in the first two years of life are tolerant of milk by 3 years of age ${ }^{4}$ and up to $80 \%$ of infants with egg allergy are tolerant of egg by 5 years of age. ${ }^{56}$ There are no similar longitudinal studies of infants with peanut allergy, and the advice that peanut allergy persists is based on a study of older children. ${ }^{1}$ The age differences between children with cows' milk or egg allergy and those with peanut allergy may account for the different rates of resolution. Follow up of a population based group of Danish children with cows' milk
Child Health, University of Southampton, Southampton SO16 6YD Jonathan O'B Hourihane, lecturer John O Warner, professor

South Manchester University Hospitals, Withington Hospital,

Manchester M20 2LR Stephen A Roberts, consultant paediatrician

Correspondence to: Dr J O'B Hourihane, Institute of Child Health, London WC1N 3EH

J.Hourihane@ich.ucl. ac.uk

BMJ 1998;316:1271-5 